\title{
Melting of Rare-Gas Crystals: Monte Carlo Simulation versus Experiments
}

\author{
V. Bocchett: ${ }^{*}$ and H. T. Diep ${ }^{\dagger}$ \\ Laboratoire de Physique Théorique et Modélisation, \\ Université de Cergy-Pontoise, CNRS, UMR 8089 \\ 2, Avenue Adolphe Chauvin, 95302 Cergy-Pontoise Cedex, France
}

(Dated: October 25, 2018)

\begin{abstract}
We study the melting transition in crystals of rare gas $\mathrm{Ar}$, Xe, and $\mathrm{Kr}$ by the use of extensive Monte Carlo simulations with the Lennard-Jones potential. The parameters of this potential have been deduced by Bernardes in 1958 from experiments of rare gas in the gaseous phase. It is amazing that the parameters of such a popular potential were not fully tested so far. In order to carry out precise tests, we have written a high-performance Monte Carlo program which allows in particular to take into account correctly the periodic boundary conditions to reduce surface effects and to reduce CPU time. Using the Bernardes parameters, we find that the melting temperature of several rare gas is from 13 to $20 \%$ higher than that obtained from experiments. We have throughout studied the case of Ar by examining both finite-size and cutoff-distance effects. In order to get a good agreement with the experimental melting temperature, we propose a modification of these parameters to describe better the melting of rare-gas crystals.
\end{abstract}

PACS numbers: 34.20.Cf, 64.70.Dv, 65.20.+w, 65.40.-b:

\section{INTRODUCTION}

Melting of crystals has always been a fascinating subject for more than a century since the discovery of the empirical Lindemann's criterion [1]. The Lindemann's criterion says that if the average of vibration amplitude $\mathrm{u}$, namely $\sqrt{\left\langle u^{2}\right\rangle}$, exceeds a certain value, usually $10 \%$ of the distance between nearest-neighbors, then the melting occurs. Times and over again, many authors have tried to find out microscopic precursor mechanisms that lead a crystal to melt. Until 30 years ago, one of the favorite pictures of melting is the softening of a phonon mode due to the temperature $(\mathrm{T})$. The atoms have no longer restoring forces which keep them staying close to their equilibrium positions: they move around and the system goes to a liquid state. The soft-mode picture has encountered some scepticism because in real crystals as well as in simulations one observes that well below the melting temperature $\left(T_{m}\right)$, many defects, dislocations, interstitial atoms ... are excited. Therefore, it is hard to believe that the system stays in a periodic structure with propagating phonon modes up to $T_{m}$. Evidence of defects is found in many works [2 [5]. Another question that is unsolved in a clear manner is the form of the potential that binds the atoms together in a given lattice structure. In a microscopic point of view, the potential should come mainly from the symmetry of atomic orbitals. But ab-initio calculations are still far away from being able to use realistic hypotheses [6]. Empirical potentials have been used instead to study melting. One can mention the popular 6-12 power Lennard-Jones (LJ) potential [7, 8], various similar power potentials, the many-body Gupta's potential [9], the Stillinger-Weber (SW) potential [10],

\footnotetext{
* virgile.bocchetti@u-cergy.fr

$\dagger$ diep@u-cergy.fr, corresponding author
}

and the Tersoff potential [11, 12]. Two-body potentials such as the LJ one crystallize atoms in the FCC at low temperatures and nothing else; this comes from the fact that LJ potential is isotropic so the atoms are crystallized in the most dense isotropic structure, namely the FCC lattice. In order to stabilize other structures, several phenomenological potentials have been introduced, often without a microscopic justification. For example, the SW potential or the Tersoff potential stabilize the diamond structure at low temperatures. These potentials have been used with success to calculate properties of $\mathrm{Si}$ clusters [13] and amorphous Si crystals [14].

In this paper, we use the LJ potential to study the melting of rare-gas crystals which have the FCC lattice structure at low temperatures. It is amazing that such a simple question was not studied with precision so far in spite of an abundance of experimental data on rare gas such as Ar, Xe, Ne and Kr. Most of the melting studies concerning rare gas were done in particular cases: small clusters [14], adlayers on a substrate, etc. The main reason to avoid to study the bulk melting may be due to some technical difficulties such as periodic boundary conditions, volume expansion with temperature, etc. Previous Monte Carlo (MC) studies of bulk melting have been carried out with LJ potential but emphasize was put on the melting mechanism rather than on the precise melting temperature in real materials $[2,3]$.

The purpose of this paper is therefore to test whether or not the experimental $T_{m}$ can be reproduced by MC simulation using the values of the LJ parameters deduced for rare gas in the gaseous state long time ago[15]. We will show here that by appropriate choices of technical procedures, we are able to obtain melting temperature for various rare gas directly from our simulations, unlike previous simulations [16-19] which have had recourse to various means and some thermodynamic functions to deduce it. We find in this work the melting temperatures for several rare gas higher than experimental values. A 
revision of the values of LJ parameters widely used in the literature for more than 50 years should be made in order to better describe the solid state of rare gas. Note that in a recent work [20], a hypothetical thermodynamic integration path is used to find the relative free energies of the solid and liquid phases, for various system sizes, at constant cutoff radius, in an attempt to explain the overestimate of the melting temperature with the LJ potential. However, due to various approximations, several results were not physically clear, in particular why the melting temperature oscillates with increasing cutoff distance.

Section $\amalg$ is devoted to a description of the model and our MC technique. The results are shown and discussed in Sect. III. Concluding remarks are given in Sect. IV]

\section{MODEL AND METHOD}

The LJ potential is given by

$$
U=\frac{1}{2} \sum_{i \neq j} V_{i j}
$$

with

$$
V_{i j}=4 \epsilon\left[a\left(\frac{\sigma}{r_{i j}}\right)^{12}-b\left(\frac{\sigma}{r_{i j}}\right)^{6}\right]
$$

where $\epsilon, a, b$ and $\sigma$ are constants, $r_{i j}$ denotes the distance between two atoms at $\mathbf{r}_{i}$ and $\mathbf{r}_{j}$. Here $a=1$ and $b=1$. We list for convenience the values of the above constants for various rare gas and their melting temperatures, using the data from Ref. [8]. Note that the constants $\epsilon$ and $\sigma$ are those deduced with some approximations using experimental data of the gaseous phase of rare gas which have been described in the pioneer paper of Bernardes [15]. The listed values of the constants should be therefore viewed as approximate values. To our knowledge, there were not papers using these constants to verify if the melting temperature is correctly obtained, either by theoretical calculations or by simulations. The absence of works dealing with this point has motivated our present work.

In one of our previous works [ $[3]$, we have used the LJ potential to investigate the mechanism which initiates the melting. We have counted the number of defects created with increasing temperature $(T)$ and found that the melting occurs when these defects interact with each other forming a kind of chains of defects which break the solid periodic state at a temperature well below the melting point. In that work, we did not use the constants of the LJ potential for any specific material. We used instead the reduced temperature and dimensionless parameters. It was not our purpose to clarify the value of melting temperature for a given kind of crystal. It is now the time to verify those constants to see whether or not they yield the correct value of $T_{m}$ for the melting of the solid phase of rare gas.

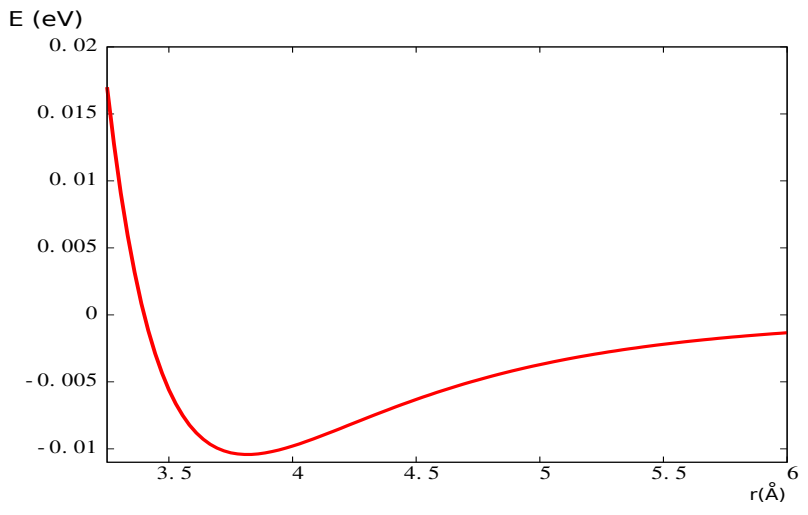

Figure 1. (Color online) The Lennard-Jones potential for Ar with the parameters listed in Table 【]

Table I. Lenard-Jones parameters

\begin{tabular}{llll}
\hline \hline Element & $\epsilon(\mathrm{eV})$ & $\sigma(\AA)$ & $T_{m}(\mathrm{~K})$ \\
\hline $\mathrm{Ar}$ & 0.01042332126 & 3.40 & 84 \\
$\mathrm{Kr}$ & 0.01404339691 & 3.65 & 117 \\
$\mathrm{Ne}$ & 0.00312075487 & 4.74 & 24 \\
$\mathrm{Xe}$ & 0.01997283116 & 3.98 & 161 \\
$\mathrm{He}$ & 0.00087381136 & 2.56 & - \\
\hline \hline
\end{tabular}

a Reference [8]

Let us describe our MC technique in the study of crystal melting. In our simulations we take a system of FCC lattice of size $N=4 \times L \times L \times L$ where $L=4,5,6,8$ and 10 namely a system of $N=256,500,1024,2048$ and 4000 atoms. Unlike spin systems on lattice where the system volume does not change with $T$, a system of vibrating atoms expands its volume with increasing $T$. We use the method described in Ref. [21] but with a modification that the displacement of an atom $i$ from its current position $\mathbf{r}_{i}$ is taken in a sphere of very small radius $r$ with two arbitrary angles $(\theta, \phi)$. To respect the uniform spatial distribution around $\mathbf{r}_{i}$, we use the Jacobian, namely we take an arbitrary $\cos (\theta)$ between -1 and +1 , instead of an arbitrary $\theta . \phi$ is taken at random between 0 and $2 \pi$. Note that we first move all atoms to new positions, and change the system volume for a small arbitrary amount, then calculate the new system energy. We tune the magnitude of displacement $(r)$ of the atoms and the volume variation in order to have an acceptation rate of displacement between $30 \%$ and $50 \%$. This criterion is empiric but it is frequently used in MC simulation. A MC step consists of moving all atoms, each with an arbitrary displacement, and changing the system volume once. The transition probability to the new state is $\exp \left(-\frac{W}{k_{B} T}\right)$ where

$$
\begin{array}{r}
W=P\left(V_{\text {new }}-V_{\text {old }}\right)+\frac{1}{2}\left(U_{\text {new }}-U_{\text {old }}\right)+ \\
N k_{B} T \ln \left(\frac{V_{\text {old }}}{V_{\text {new }}}\right)
\end{array}
$$


where $P$ is the pressure which is set to zero here (constant pressure), $V_{\text {old }}$ and $V_{\text {new }}$ are old and new system volumes, $U_{\text {old }}$ and $U_{\text {new }}$ old and new system energies.

One of the most difficult tasks in melting simulations is the application of the periodic boundary conditions. This is due to random positions of atoms at the crystal boundaries. We have taken the following actions to shorten, without loosing accuracy, our simulation CPU time:

(i) each atom has a list of neighbors up to a distance $r_{d}$ longer than the potential cutoff distance $r_{c}$. To establish the list for the first time, we have to calculate all distances and we select neighbors at $r \leq d$. It takes time. The fact to choose $r_{d}>r_{c}$ is to ensure that for small displacements, some neighbors with $r_{c}<r<r_{d}$ can enter inside the cutoff sphere without the need to reestablish the list of neighbors

(ii) when an atom moves outside a sphere of radius $r_{1}$ around its equilibrium position, its list of neighbors is updated by recalculating all pairs, neighbors up to $r_{d}$ fill the new list. $r_{1}$ is chosen to be equal to $20 \%$ of $r_{0}$ the nearest-neighbor distance at equilibrium $(T=0)$

(iii) periodic boundary conditions are applied by translating the system in all directions in a manner that avoids mismatches at the boundaries.

The last step is the most important technically. We show in Fig. 2 a snapshot of the system and its translated atoms by periodic boundary conditions. We see that if they are of the same color, one cannot distinguish the system boundaries.

In the following, we will show results with a cutoff distance $r_{c}$ equal to twice $\ell$, the lattice constant of the FCC cell, namely $r_{c}=2 \ell=2 r_{0} \sqrt{2}$ where $r_{0}$ is the nearestneighbor distance. As seen in section IIID this value of $r_{c}$ is large enough to ensure a correct value of the melting temperature.

\section{MELTING OF RARE-GAS CRYSTALS}

\section{A. The case of Ar}

Let us show first the results for Ar obtained by using the values listed in Table 1 . We will discuss next the modification necessary for obtaining the result in agreement with experiment. In order to take a correct average of physical quantities, we record spontaneous values of all physical quantities during each $\mathrm{MC}$ run. We have to go to several millions of MC steps before observing statistical fluctuations around equilibrium. We show an example of the energy per atom $E$ versus MC time in Fig. 3 for $N=256$ atoms at two temperatures $T=92 \mathrm{~K}$ and 94 $\mathrm{K}$. At $T=92 \mathrm{~K}$, the system is still in the solid phase. Its energy is stabilized after about one million MC steps per atom. However, at $T=94 \mathrm{~K}$, the system melts after three millions of MC steps per atom: $E$ is stabilized in the liquid phase only after such a long $\mathrm{MC}$ time. It is very important to emphasize that the convergence time

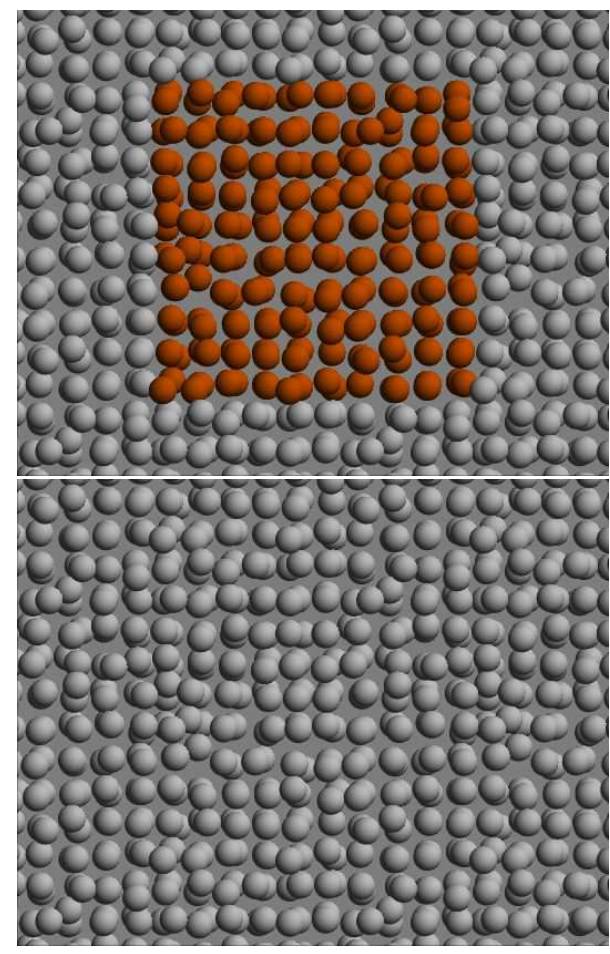

Figure 2. (Color online) Upper: Snapshot of the system (magenta) with its translated atoms (gray) for periodic boundary conditions, Lower: The same snapshot of the system with its translated atoms but with the same color (online): boundaries are undistinguishable. Argon crystal at $T=95 \mathrm{~K}$ with $N=500$ atoms.

to equilibrium depends on various MC parameters such as value of displacement magnitude $r$, volume variation $\delta$ etc. So, nothing can replace an observation of the timedependence of physical parameters during the simulation, although this consumes a huge computer memory.

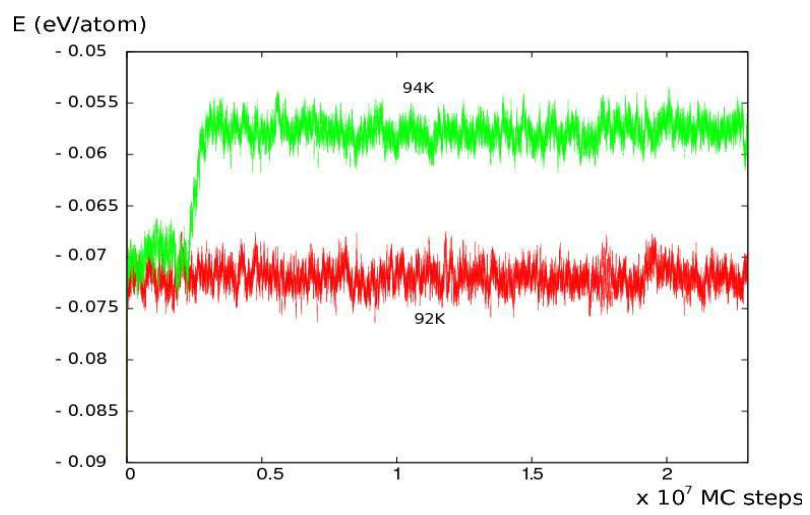

Figure 3. (Color online) Energy per atom $E$ versus number of MC steps per atom $t$ for an Argon crystal at $T=92 \mathrm{~K}$ (red) and $T=94 \mathrm{~K}$ (green) with $N=256$ atoms.

We show in Fig. 4 the energy per atom $E$ versus $T$ in the case of Ar with $N=256$ atoms, using the parameters given in Table 1 . We observe here that the melting occurs 
at $T_{m} \simeq 93 \mathrm{~K}$ with a large latent heat. This value of $T_{m}$ is rather far from the experimental data given in Table [1] We return to this point later. We show now the snapshots of the system for different temperatures in Fig. [5] below and above the melting. As seen, the system just starts to be spatially disordered at $92 \mathrm{~K}$, and is well in the disordered phase (liquid) at $100 \mathrm{~K}$.

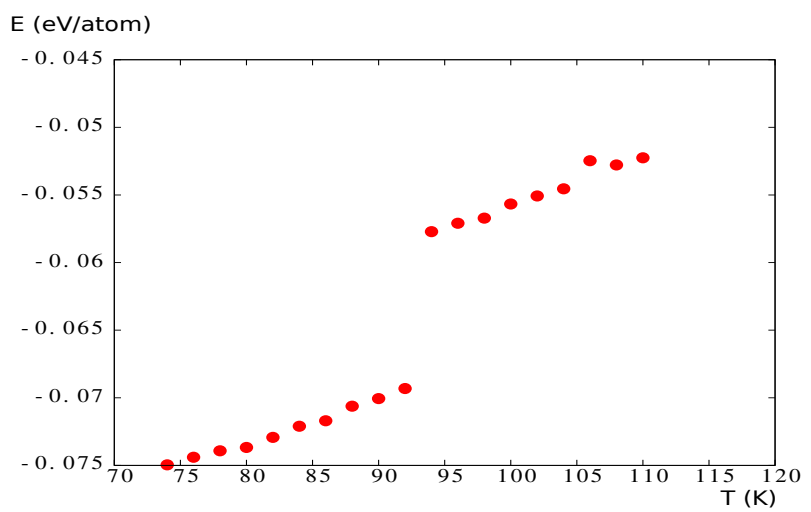

Figure 4. (Color online) Energy per atom $E$ versus temperature $T$ for an Argon crystal of 256 atoms, obtained with the parameters listed in Table I. One observes that the melting temperature is $T_{m} \simeq 93 \mathrm{~K}$ while the experimental value is 84 K. Run of 50 millions MC steps/atom. See text for comments.
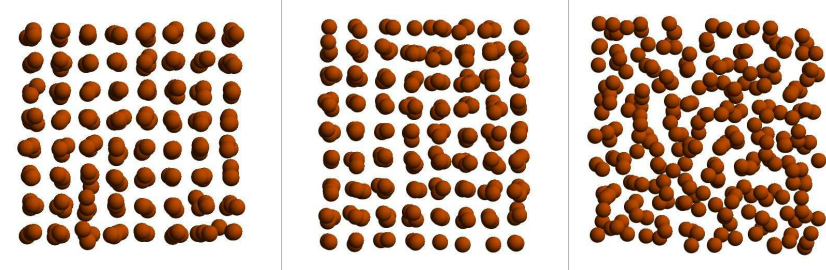

Figure 5. (Color online)Instantaneous pictures of the supercell of Ar for different temperatures, respectively from left to right : $75 \mathrm{~K}$ (far below $T_{m}$ ), $92 \mathrm{~K}$ (close to $T_{m}$ ) and $100 \mathrm{~K}$ (above $T_{m}$ ).

The radial distribution for $\mathrm{Ar}$ is shown in Fig. [6 at several temperatures. One sees clearly the peaks at first, second and third neighbor distances for $T<T_{m}$ indicating the crystalline phase, while there is no such clear distinction for $T>T_{m}$ where a liquid phase is set in.

We display in Fig. 7 the curve which shows the dilatation of the simulation box against the temperature.

Let us discuss now the difference between our value of $T_{m}(N=256)=93 \mathrm{~K}$ with the experimental value $T_{m}(\exp )=84 \mathrm{~K}$. Clearly, the parameters given in Table [1] which have been deduced with experimental data for gaseous Ar do not describe well the melting of solid Ar. Note however that the above value of $T_{m}$ is for $N=256$ atoms. If we consider larger samples, $T_{m}$ should increase further.

In order to modify correctly the LJ parameters while respecting the known properties of Ar, we use the listed

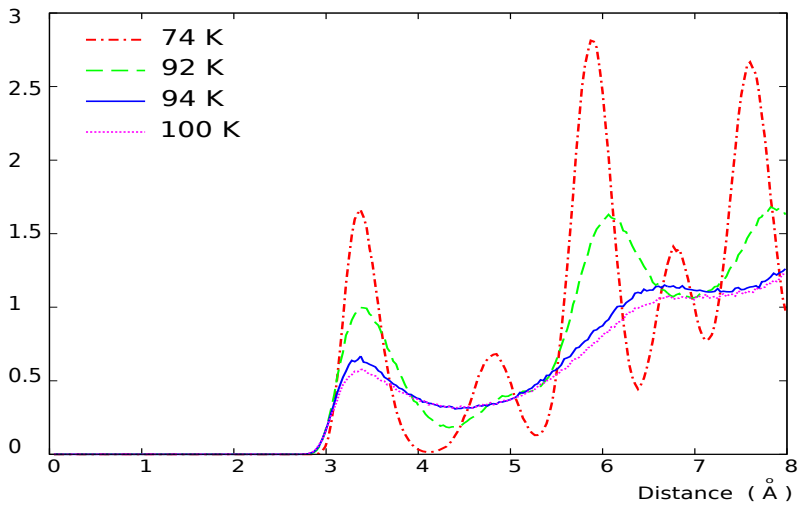

Figure 6. (Color online) Radial distribution of Ar for different temperatures: below melting $T=74 \mathrm{~K}$ (discontinued red) and $92 \mathrm{~K}$ (discontinued green), above melting $T=94 \mathrm{~K}$ (solid blue) and $100 \mathrm{~K}$ (solid magenta), with $N=256$ atoms.

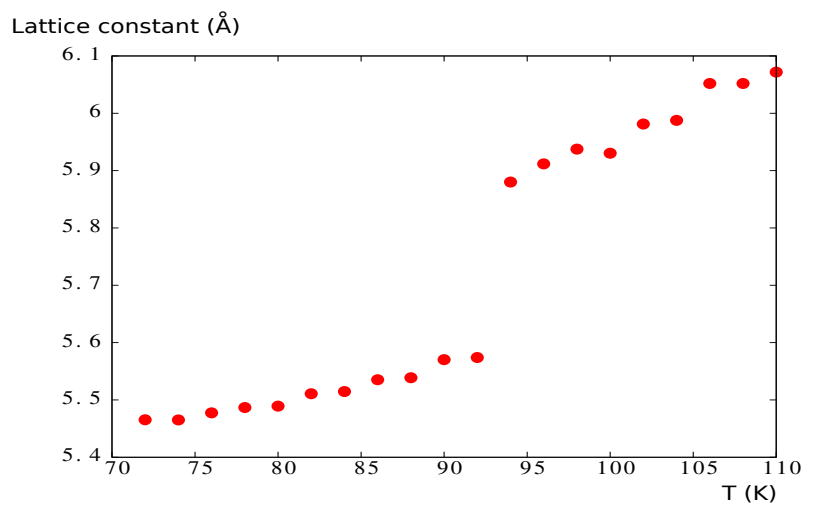

Figure 7. (Color online)Evolution of the lattice constant against temperature $T$. As we can see, there is a jump of this quantity which occurs at the same temperature of the jump of the energy in Fig. 4 Argon crystal, $N=256$.

values of LJ parameters to estimate the size effect, namely to find the value of $T_{m}(N=\infty)$ for an infinite crystal. Once this task is done, we then we look for the parameters $(\epsilon, \sigma)$ which give correctly the experimental $T_{m}(\exp )$. The effect of the cutoff distance will be examined.

\section{B. Size effect}

The size effect is an important fact when we work with numerical simulations because of the finite size of the simulation cell. In second-order phase transitions, finitesize scaling laws allow us to calculate the critical exponents by varying the system size [22 25]. The correlation length is infinite at the critical point in the thermodynamic limit. However, in first-order transitions, the correlation length is finite at the transition point, the two phases coexist [26, 27] and the energy is discontinuous. The correlation is finite at the transition temperature means that when the system size exceeds the correlation 
length the transition temperature does not depend anymore on the system size. We need not to go to the infinite limit to find saturated transition temperature. Note that if the size of the system is too small, a first-order phase transition can appear as a second-order phase transition if the correlation length exceeds the system size. As we can see in Fig, 8, fortunately when the size of the simulation cell is larger than 5 lattice constants, melting temperature $T_{m}$ is bounded by $102 \mathrm{~K}$. It means that the correlation length at the melting temperature is smaller than 5 lattice spacings.

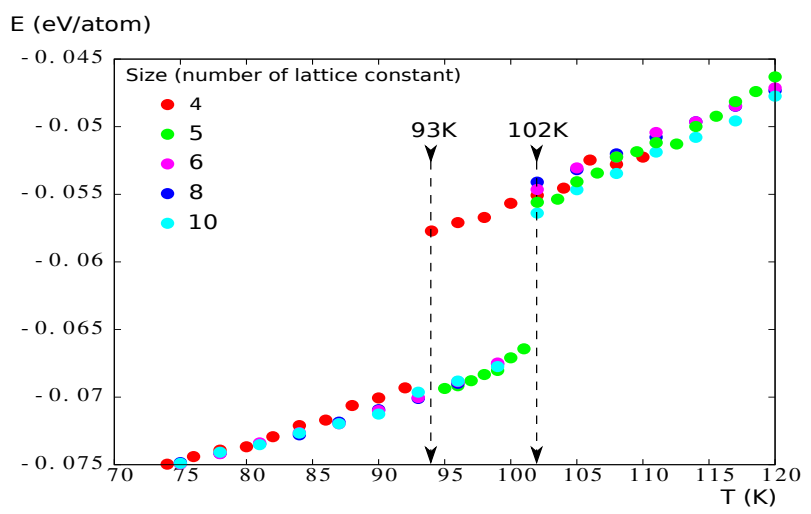

Figure 8. (Color online)Energy per atom versus $T$ with various system sizes from $N=256$ (4 FCC lattice cells) to $N=4000$ (10 FCC lattice cells). The arrows indicate $T_{m}$ for the smallest and largest sizes. See text for comments.

The saturated melting temperature obtained by our MC simulations is thus $18 \mathrm{~K}$ (or 20\%) higher than the experimental one. This disagreement come from the values of the Bernardes parameters used for the LJ potential. The reason is that these values have not been calculated by fitting with the melting temperature but they were calculated with a low-density gas using the second virial coefficient [15]. The Bernardes parameters are therefore questionable as we can see in Refs. 28 30]. Taken from those articles, the values of the nearest-neighbor distance, the cohesive energy and the bulk modulus calculated using the Bernardes parameters are listed in Table III. We can see the differences between experimental values and theoretical ones for all cases $\mathrm{Ne}, \mathrm{Ar}, \mathrm{Kr}$ and Xe. The Bernardes parameters also yield the high melting temperature found in our simulations with respect to the experimental one.

At this stage, it is interesting to note that Molecular Dynamics (MD) simulation of melting of a perfect crystal with periodic boundary conditions produce superheating. There is an empirical rule which states that the melting temperature of a crystal without any surface and any defects, is $20 \%$ higher than the true thermodynamic melting temperature $T_{m}$. However in MC simulations, defects and dislocations are naturally created in the crystal by means of random numbers used in every MC step for atom displacements. Thus, the superheating should not exist. Agrawal et al. have shown in Ref. [31] that for
Table II. Experimental (Exp.) and theoretical (Th.) values of NN distance $r_{0}$, cohesive energy $u_{0}$ and bulk modulus $B_{0}{ }^{a}$ for comparison. See text for comments.

\begin{tabular}{llllll}
\hline \hline & & $\mathrm{Ne}$ & $\mathrm{Ar}$ & $\mathrm{Kr}$ & $\mathrm{Xe}$ \\
\hline$r_{0}$ & Exp. & 3.13 & 3.75 & 3.99 & 4.33 \\
& Th. & 2.99 & 3.71 & 3.98 & 4.34 \\
\hline$u_{0}$ & Exp. & -0.02 & -0.08 & -0.11 & -0.17 \\
& Th. & -0.027 & -0.0889 & -0.12 & -0.172 \\
\hline$B_{0}$ & Exp. & 1.1 & 2.7 & 3.5 & 3.6 \\
& Th. & 1.81 & 3.18 & 3.46 & 3.81 \\
\hline \hline
\end{tabular}

${ }^{a}$ Reference [28 30]

Ar, with MC simulation, $T_{m}$ is about $15 \%$ higher than the experimental value. With our results for Ar, we find an increase of about $20 \%$. For $\mathrm{Kr}$, we find an increase of $13 \%$ for $N=256$ atoms after $23 \times 10^{6} \mathrm{MC}$ steps per atom. This increase is more important if we consider larger sizes as seen in the case of Ar. As said, the high values of $T_{m}$ in MC simulations are not due to the superheating as in MD simulations. Rather, we believe that these high values are due to the inaccuracy of the listed Bernardes parameters. We will propose a modification in the following

\section{Modification of Lennard-Jones parameters}

In order to reduce the $T_{m}$ value, we propose now to modify the value of $\epsilon$, the prefactor of the LJ potential, and the coefficient $\sigma$.

Note that in papers dealing with melting in other materials by means of MD calculations or MC simulations, there have been several propositions to modify constants appearing in potentials in order to obtain a correct agreement with experimental value of $T_{m}$. This is because these constants are often deduced from experimental data which are not valid for the whole temperature range. Among these papers, we can mention the case of melting of Si crystal studied by MD [32, 33] and MC simulations [31, 34], using the Tersoff potential [11]. Our proposition to modify some constants of the LJ potential when applied to a rare-gas crystal is certainly a necessity in order to reproduce the experimental $T_{m}$.

We have done simulations with different pairs of $(\epsilon, \sigma)$. It turned out that $T_{m}$ depends essentially on $\epsilon$. There is however an optimal value of $\sigma$ which is $\sigma=3.44 \AA$ corresponding to the experimental nearest-neighbor distance $r_{0}=3.75 \AA$ of Ar (cf. Table II). We show in Fig. 9 the curves obtained for two selected values of $\epsilon=0.008767853$ and 0.008951794 which give respectively $T_{m}=83 \mathrm{~K}$ and $86 \mathrm{~K}$. These values of $T_{m}$ are in agreement with the experimental value $84 \mathrm{~K}$ within statistical errors. Note that the modified $\epsilon$ is about $15 \%$ smaller than the original Bernardes value $\epsilon=0.01042332126$. 


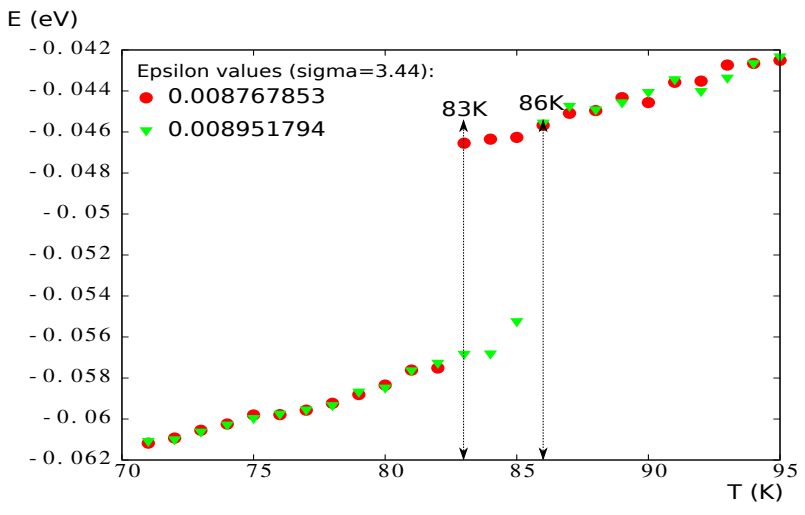

Figure 9. (Color online) Energy per atom versus $T$ for two selected values of $\epsilon$, with $\sigma=3.44 \AA$, for an Ar crystal with $N=500$. The arrows indicate $T_{m}$ for the two indicated values of $\epsilon$. See text for comments.

\section{Effect of cutoff distance}

At this stage, a natural question we ask ourselves is "what is the effect of the cutoff distance $r_{c}$ ?". We know that for a long-range interaction, the longer the interaction range is the lower the energy becomes. As a consequence, the melting transition is higher. However, as $r_{c}$ increases, the contribution of neglected neighbors becomes smaller. From a certain value of $r_{c}, T_{m}$ does not vary significantly. This is observed in Fig. 10 where $T_{m}$ is saturated for $r_{c} \geq 2 \ell$, i. e. $r_{c} \gtrsim 10.6 \AA$. All the results shown above for $r_{c}$ are valid in the discussion of the size effect and the modification of $\epsilon$ and $\sigma$.

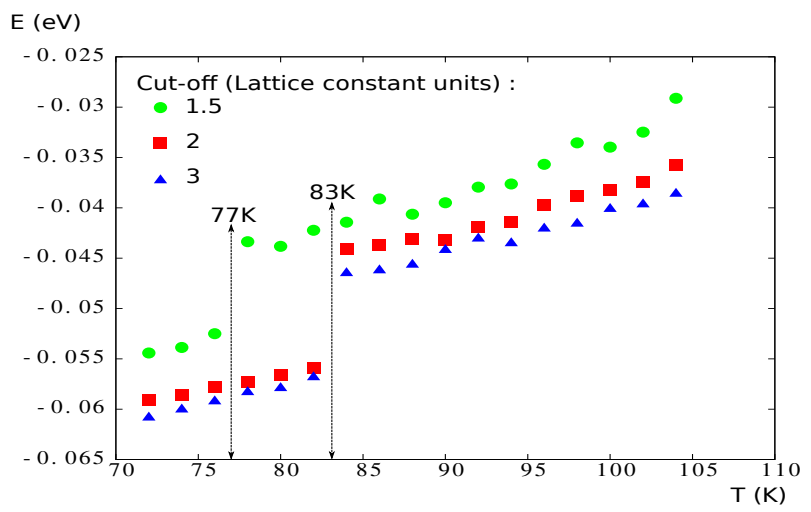

Figure 10. (Color online) Energy per atom versus $T$ with various values of $r_{c}$ for Ar crystal with $N=500$. The left arrow indicates $T_{m}$ for $r_{c}=1.5 \ell$ and the right arrow indicates $T_{m}$ for $r_{c}=2 \ell \sim 10.6 \AA$ and $3 \ell \sim 15.9 \AA$. Note that $\ell$ is the FCC lattice constant which is equal to $r_{0} \sqrt{2}$ where $r_{0}=3.75$ $\AA$ is the NN distance. See text for comments.

\section{E. The case of other rare gases}

In order to show that our algorithm works well with other rare gas, we have plotted the curve of energy versus temperature, obtained for Krypton and Xenon in Fig. 11. Again here, we see that $T_{m}$, even for a small size, is already higher than the experimental value for each crystal. We think that the Bernardes parameters for $\mathrm{Kr}$ and Xe should be modified to get an agreement with experiments as what proposed above for Ar.

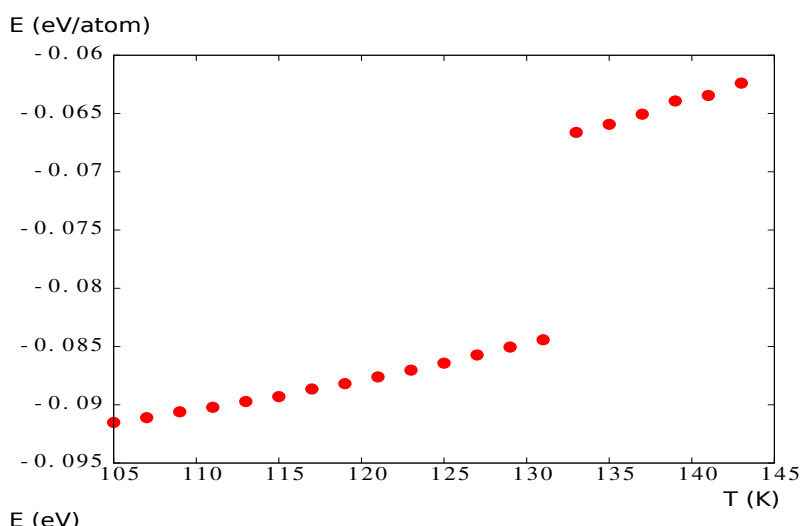

$\mathrm{E}(\mathrm{eV})$

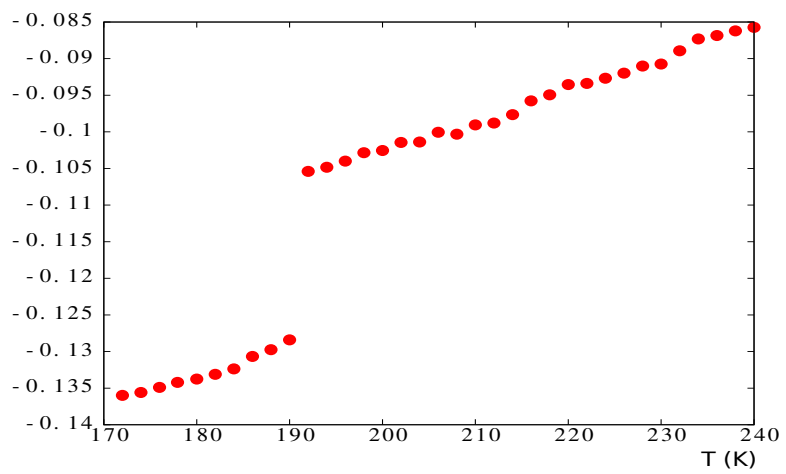

Figure 11. (Color online) Upper: Energy versus temperature for a Krypton perfect crystal with $N=256$ atoms. One observes that $T_{m}=132 \mathrm{~K}$ while the experimental value is 117 K. Lower: Energy versus temperature for a Xenon perfect crystal with $N=500$ atoms. One observes that $T_{m}=191$ $\mathrm{K}$ while the experimental value is $161 \mathrm{~K}$. These curves have been obtained with the Bernardes values of parameters.

\section{CONCLUSION}

We have shown in this paper results of the melting temperature for rare gas, by performing extensive $\mathrm{MC}$ simulations with the LJ potential. We have obtained directly from our simulations physical quantities such as internal energy, lattice constant and radial distribution as functions of temperature. We have shown that melting occurs with a large latent heat and a jump in the lattice constant. Effects of system size and cutoff distance have been investigated. Let us emphasize that the theoretical LJ parameters widely used in the literature [15] yield a 
melting temperature higher than the experimental one as seen above, from $\sim 15 \%$ for $\mathrm{Kr}$ to $\sim 20 \%$ for Ar. This is not a surprise because those LJ parameters already yield theoretical NN distance, cohesive energy and bulk modulus different from corresponding experimental ones (see Table II). We have demonstrated that, in order to reduce the melting temperature to fit with experiments, it is necessary to modify the original Bernardes LJ parameter $\epsilon$ in such a way to reduce the energy at $T=0$.
The effect of $\sigma$, within possible values of NN distance, is very small on $T_{m}$. A good agreement on $T_{m}$ between experiments and simulations for Ar is obtained with the modified values given in Fig. 9. For other rare gas such as $\mathrm{Kr}$ and $\mathrm{Xe}$, we have to proceed to a modification of their Barnardes parameters in a manner similar to that done above for Ar if we want to get a good agreement with experiments.
[1] F. A. Lindemann, Phys. Z 11, 609 (1910).

[2] Liliana Gómez, A. Dobry and H. T. Diep, Phys. Rev. B 63, 224103 (2001).

[3] Liliana Gómez, A. Dobry, Ch. Geuting, H. T. Diep, and L. Burakovsky, Phys. Rev. Lett. 90, 095701 (2003).

[4] N. D. Mermin, Phys. Rev. 176, 250 (1968).

[5] D. R. Nelson and B. I. Halperin, Phys. Rev. B 19,... (1979).

[6] D. Pettifor, Bonding and Structure of Molecules and Solids, Oxford University Press, London (1995).

[7] N. W. Aschrof and N. D. Mermin, Solid State Physics, Saunders, Philadelphia (1976).

[8] Ch. Kittel, Introduction to Solid State Physics, 8-th edition, John Wiley \& Sons, New York (2008).

[9] R. P. Gupta, Phys. Rev. B 23, 6265-1 (1981).

[10] F. H. Stillinger and T. A. Weber, Phys. Rev. B 31, 5262 (1985).

[11] J. Tersoff, Phys. Rev. Lett. 56, 632 (1986).

[12] J. Tersoff, Phys. Rev. B 39, 5566 (1989).

[13] P. T. Dinda, G. Vlastou-Tsinganos, N. Flytzanis, and A. D. Mistriotis, Phys. Rev. B 51, 13697 (1995).

[14] R. L. C. Vink, G. T. Barkema, W. F. Van-der-Weg, and N. Mousseau, J. of Non-Crystalline Solids, 282, 248 (2001).

[15] N. Bernardes, Phys. Rev. 112, 1534 (1958).

[16] R. Morris and X. Song, J. Chem. Phys. 116, 9352 (2002).

[17] J. R. Errington, J. Chem. Phys. 120, 3130 (2004).

[18] E. A. Mastny and J. J. de Pablo, J. Chem. Phys. 122, 124109 (2005).

[19] G. C. McNeil-Watson and N. Wilding, J. Chem. Phys. 124, 064504 (2006).
[20] E. A. Matsny and J. J. de Pablo, J. Chem. Phys. 127, 104504 (2007).

[21] M. P. Allen and D. J. Tildesley, Computer simulation of liquids, Oxford Univ., Clarendon Press, London (1987).

[22] D. P. Laudau and K. Binder, in Monte Carlo Simulation in Statistical Physics, Ed. K. Binder and D. W. Heermann, Springer-Verlag, New York (1988).

[23] P. C. Hohenberg and B. I. Halperin, Rev. Mod. Phys. 49 435 (1977).

[24] A. M. Ferrenberg and R. H. Swendsen, Phys. Rev. Lett. 61, 2635 (1988) ; ibid 63, 1195(1989).

[25] A. M. Ferrenberg and D. P. Landau, Phys. Rev. B44, 5081 (1991).

[26] M. N. Barber, in Phase Transitions and Critical Phenomena, vol. 8, ed. C Domb and J. L. Lebowitz, p. 145, Academic, New York (1983).

[27] K. Binder, Rep. Prog. Phys. 50, 783-859 (1987).

[28] M. L. Klein, G. K. Horton, and J. L. Feldman, Phys. Rev. 184, 968 (1969).

[29] D. N. Batchelder, D.L. Losee, R. O. Simmons, Phys. Rev. 162, 767 (1967).

[30] E. R. Bobbs and G. O. Jones, Report on Progress in Physics 20, 516 (1957).

[31] P. Agrawal, L. Raff and R. Komanduri, Phys. Rev. B 72, 125206 (2005).

[32] S. J. Cook and P. Clancy, Phys. Rev. B 47, 7686 (1993).

[33] S. Yoo, X. C. Zeng, and J. R. Morris, J. Chem. Phys. 120, 1654 (2004).

[34] V. Bocchetti, H. T. Diep, H. Enriquez, H. Oughaddou and A. Kara, in preparation. 